\title{
Implicit Contextual Learning in Prodromal and Early Stage Huntington's Disease Patients
}

\author{
Marieke van Asselen, ${ }^{1}$ Inês Almeida, ${ }^{1}$ Filipa Júlio, ${ }^{1,2}$ Cristina Januário, ${ }^{2}$ Elzbieta Bobrowicz Campos, ${ }^{3}$ \\ Mário Simões, ${ }^{3}$ AND Miguel Castelo-Branco ${ }^{1}$ \\ ${ }^{1}$ Visual Neuroscience Laboratory, IBILI, Faculty of Medicine, University of Coimbra, Portugal \\ ${ }^{2}$ Department of Neurology, University Hospital Coimbra, Portugal \\ ${ }^{3}$ Faculty of Psychology and Educational Sciences, University of Coimbra, Portugal
}

(Received October 27, 2011; Final Revision February 9, 2012; Accepted February 9, 2012)

\begin{abstract}
Huntington's disease (HD) is a genetic neurodegenerative disorder affecting the basal ganglia. These subcortical structures are particularly important for motor functions, response selection and implicit learning. In the current study, we have assessed prodromal and symptomatic HD participants with an implicit contextual learning task that is not based on motor learning, but on a purely visual implicit learning mechanism. We used an implicit contextual learning task in which subjects need to locate a target among several distractors. In half of the trials, the positions of the distractors and target stimuli were repeated. By memorizing this contextual information, attention can be guided faster to the target stimulus. Nine symptomatic HD participants, 16 prodromal HD participants and 22 control subjects were included. We found that the responses of the control subjects were faster for the repeated trials than for the new trials, indicating that their visual search was facilitated when repeated contextual information was present. In contrast, no difference in response times between the repeated and new trials was found for the symptomatic and prodromal HD participants. The results of the current study indicate that both prodromal and symptomatic HD participants are impaired on an implicit contextual learning task. (JINS, 2012, 18, 689-696)
\end{abstract}

Keywords: Huntington's disease, neurodegenerative disorder, implicit contextual learning, basal ganglia, visual search

\section{INTRODUCTION}

Huntington's disease (HD) is a neurodegenerative disease, caused by a CAG repeat expansion leading to an abnormal huntingtin protein. Damage is particularly found in the striatum, although other regions of the brain are affected as well, including cortical areas. The most characteristic symptoms of HD are motor deficits, including chorea, rigidity, and abnormal posturing. In addition to motor symptoms, cognitive and behavioral changes are generally also found, such as memory and executive dysfunctions, depression, apathy and other psychiatric manifestations. Clinical diagnosis is based on motor symptoms. However, since HD is a disorder with a known genetic etiology, (Huntington's Disease Collaborative Research Group, 1993), people that are at risk of having the disease can be identified before onset of the first symptoms, allowing for directed search of preclinical deficits. This has indicated that subtle cognitive impairments can be found in prodromal

Correspondence and reprint requests to: $\mathrm{M}$. van Asselen, IBILI-Faculdade de Medicina, Azinhaga de Santa Comba-Celas, 3000-354 Coimbra. E-mail: masselen@fmed.uc.pt
HD gene carriers, in particular affecting memory, executive functioning and psychomotor speed (e.g., Farrow et al., 2007; Kirkwood et al., 1999, 2000; Lawrence et al., 1998; Lemier, Decruyenaere, Evers-Kieboms, Vandenbussche, \& Dom, 2004; Robins Wahlin, Lundin, \& Dear, 2007; Snowden, Craufurd, Thompson, \& Neary, 2002; Stout et al., 2011; Tabrizi et al., 2009, 2011). Furthermore, on a neural level, damage to the striatum is already found in prodromal HD gene carriers and some studies have reported subtle cortical abnormalities as well (e.g., Gómez-Ansón et al., 2009; Harris et al., 1999; Nopoulos et al., 2010; Paulsen et al., 2010; Tabrizi et al., 2009, 2011; Thieben et al., 2002). The striatum (including the caudate nucleus and putamen) is a part of the basal ganglia, which are a group of subcortical nuclei that are important for executive and motor functions, but are also involved in learning mechanisms. Prodromal HD patients have been found to be impaired on tasks that are dependent on the striatum, in particular implicit learning tasks, such as motor pursuit (Heindel, Salmon, Shults, Walicke, \& Butters, 1989), rotary pursuit (Gabrieli, Stebbins, Singh, Willingham, \& Goetz, 1997), mirror reading, and implicit sequence learning (Ghilardi et al., 2008; Kim et al., 2004). 
Implicit contextual learning is a type of implicit learning that has received much attention over the past decade. It is based on the notion that our environment contains a large amount of contextual information that characterizes specific environments. By memorizing this contextual information we can facilitate a visual search through our environment, which underlies a top-down attention guiding mechanism (e.g., Chun \& Jiang, 1998; Chun, 2000). Interestingly, this mechanism does not require conscious attention, and, therefore, is an implicit learning process. Furthermore, although the task requires a motor response to measure response times, the learning mechanism is purely based on the association between visual information.

It was repeatedly shown that the medial temporal lobes are involved in implicit contextual learning (Chun \& Phelps, 1999; Greene, Gross, Elsinger, \& Rao, 2007; Manns \& Squire, 2001; Preston \& Gabrieli, 2008). The medial temporal lobes, in particular the hippocampus, are important for a range of memory processes, such as explicit memory and binding of features in memory. Although the importance of the MTL in implicit contextual learning is supported by several studies, we showed in a recent study that patients with Parkinson's disease were unable to learn contextual information during an implicit contextual cueing task (Van Asselen et al., 2009). Parkinson's disease is a neurodegenerative disease in which dopamine cells in the substantia nigra degenerate. Since the substantia nigra is the main source of dopamine to the striatum, the latter is also affected. Importantly, in the study of Van Asselen et al. (2009) patients were tested in an early stage of the disease to ensure damage was largely limited to the basal ganglia. This is because, although Parkinson's disease affects the basal ganglia early on, the frontal cortex is also affected in later stages of the disease. Together, this suggests a critical network underlying implicit contextual learning, including both the medial temporal lobes and the basal ganglia.

In the current study, we aimed to investigate implicit contextual learning in Huntington's disease. To define whether an impairment can already be found before onset of the first clinical features, we included both prodromal and symptomatic HD participants. If the basal ganglia are indeed involved in implicit contextual learning, we expect to find no learning effect in the symptomatic HD patients, in contrast to the healthy control subjects. Considering the fact that damage to the basal ganglia is already found in prodromal HD patients, impairment is also expected in this group.

\section{METHODS}

\section{Participants}

Two patient groups were included in this study: 16 prodromal Huntington's disease participants and 9 symptomatic Huntington's disease participants. All patients were seen by an experienced neurologist (C.J.), who used the motor scale of the Unified Huntington Disease Rating Scale (UHDRS) for clinical diagnosis. Genetic testing was used in all patients to define the number of CAG repeats (see Table 1). Furthermore, we tested 22 healthy control subjects. Participants were excluded from the study if they had fewer than 4 years of education or when they had neurological or psychiatric disorders that were not related to HD. The three groups were matched for education level $[F(2,44)=2.1 ; p=.13]$. One-way analysis of variance (ANOVA) demonstrated an overall difference between the ages of the three groups of participants $[F(2,44)=4.3 ; p<.05]$. However, post-hoc analyses indicated no age difference between the controls and prodromal HD participants and the controls and symptomatic HD participants. A significant difference was found, as expected, between the prodromal and symptomatic HD participants. Considering the progressive nature of the disease, this difference was unavoidable. Characteristics of these groups are presented in Table 1.

\section{Neuropsychological Testing}

All participants were tested with an extensive neuropsychological test battery to define whether prodromal and symptomatic HD participants had other cognitive deficits. We used the 12-item short form of the Raven Advanced Progressive Matrices (Raven, Raven, \& Court, 1993) and the Vocabulary subtest of the WAIS-III (Wechsler, 1997, 2008) as an indication of intelligence. Visual perception was tested with the Rey Complex Figure Test (Rey, 1941; Osterrieth, 1944), the Benton Visual Retention test (Benton, 1974) and the Benton Visual Form Discrimination test (Benton, Hamsher, Varney, \& Spreen, 1983). Verbal memory was tested using a Portuguese version of the Rey Auditory Verbal Learning Test (Cavaco et al., 2008; Rey, 1964). To assess working memory and executive functioning we used the Trail Making test and Digit Symbol subtest of the WAIS-III and the Corsi BlockTapping task (Berch, Krikorian, \& Huha, 1998; Kessels, Van Zandvoort, Postma, Kappelle, \& De Haan, 2000). For the latter task, the product of the total number of correct trials and the length of the largest sequence was calculated (Kessels et al., 2000). The Dementia Rating Scale-2 (Mattis, Jurica, \& Leitten, 2002) and the Beck Depression Inventory (Beck, Ward, Mendelson, Mock, \& Erbaugh, 1961; Portuguese adaptation: Vaz Serra \& Pio Abreu, 1973a, 1973b) were used to test for dementia and depression subsequently. Handedness was defined by using a translated version of the Edinburgh Handedness Inventory (Oldfield, 1971). Informed consent was obtained according to the Declaration of Helsinki and all procedures were approved by our local ethics committee.

\section{Implicit Contextual Cueing Task}

Subjects were tested with an implicit contextual cueing task in which spatial information was used as a contextual cue (Van Asselen et al., 2009). This task was programmed using the Presentation software (Neurobehavioral systems) and displayed on a computer screen. The implicit contextual cueing task (Figure 1) is a visual search task in which subjects 
Table 1. Characteristics of the prodromal and symptomatic HD participants and the control group

\begin{tabular}{|c|c|c|c|}
\hline & Controls & Prodromal HD & Symptomatic HD \\
\hline & Mean (SE) & Mean (SE) & Mean (SE) \\
\hline Age (years) & $41.0(2.3)$ & $36.2(1.8)$ & $48.8(4.6)^{3}$ \\
\hline Education (years) & $11.4(0.9)$ & $9.9(1.0)$ & $8.1(1.3)$ \\
\hline Gender (F:M) & $13: 9$ & $14: 2$ & $0: 9$ \\
\hline Handedness (R:L) & $22: 1$ & $16: 0$ & 9:0 \\
\hline Disease duration (years) & - & - & $5.3(1.5)$ \\
\hline UHDRS (motor) & - & $1.5(0.3)$ & $31.3(3.0)$ \\
\hline CAG repeats & & $42.2(0.4)$ & $44.1(0.9)$ \\
\hline Beck Depression Inventory ${ }^{\mathrm{b}}$ & $4.9(1.1)$ & $7.5(1.4)$ & $11.2(0.9)$ \\
\hline Dementia Rating Scale (total) ${ }^{a}$ & $138.0(1.2)$ & $136.3(1.6)$ & $122.6(3.0)^{2,3}$ \\
\hline Rey Complex Figure (total) ${ }^{\mathrm{a}}$ & $30.7(0.9)$ & $29.4(1.0)$ & $26.4(1.4)^{2}$ \\
\hline Digit Symbol - WAIS-III (raw score) ${ }^{\mathrm{a}}$ & $62.9(4.1)$ & $56.8(3.2)$ & $30.8(3.5)^{2,3}$ \\
\hline Auditory Verbal Learning test (total trials $1-5)^{\mathrm{a}}$ & $53.2(1.2)$ & $53.6(2.1)$ & $34.9(3.3)^{2,3}$ \\
\hline Auditory Verbal Learning test (Recall) ${ }^{\mathrm{a}}$ & $10.8(0.4)$ & $12.1(0.5)$ & $6.1(0.9)^{2,3}$ \\
\hline Auditory Verbal Learning test (Recognition) ${ }^{\mathrm{a}}$ & $29.6(0.2)$ & $29.7(0.1)$ & $26.8(1.0)^{2,3}$ \\
\hline Raven Advanced Progressive Matrices $(\max 12)^{\mathrm{a}}$ & $7.8(0.6)$ & $7.6(0.5)$ & $3.8(0.6)^{2,3}$ \\
\hline Corsi Block Tapping task (Direct) ${ }^{\mathrm{a}}$ & $48.6(3.9)$ & $46.6(3.6)$ & $31.2(2.8)^{2,3}$ \\
\hline Corsi Block Tapping task (Inverse) ${ }^{\mathrm{a}}$ & $43.6(5.5)$ & $46.1(3.7)$ & $21.7(4.2)^{2,3}$ \\
\hline Vocabulary- WAIS III (raw score) ${ }^{a}$ & $39.5(2.6)$ & $30.9(3.1)$ & $27.2(4.1)^{2}$ \\
\hline Benton Visual Retention test (total correct) ${ }^{\mathrm{a}}$ & $6.2(0.4)$ & $5.9(0.4)$ & $3.4(0.6)^{2,3}$ \\
\hline Benton Visual Retention test (errors) ${ }^{\mathrm{b}}$ & $5.9(0.7)$ & $6.3(0.8)$ & $12.3(0.9)^{2,3}$ \\
\hline Benton Visual Form Discrimination test (total correct) ${ }^{\mathrm{a}}$ & $29.7(0.5)$ & $29.8(0.6)$ & $27.3(1.3)$ \\
\hline Trail Making Test A (seconds) ${ }^{\mathrm{b}}$ & $42.0(4.7)$ & $37.3(5.5)$ & $74.6(7.3)^{2,3}$ \\
\hline Trail Making Test B (seconds) ${ }^{\mathrm{b}}$ & $78.1(13.6)$ & $75.8(15.9)$ & $215.9(21.2)^{2,3}$ \\
\hline
\end{tabular}

Note. UHDRS $=$ Unified Huntington Disease Rating Scale; WAIS-III $=$ Wechsler Adult Intelligence Scale-Third edition. $1=$ Significant difference $(p<.05)$ between the controls and prodromal HD group; $2=$ Significant difference $(p<.05)$ between the controls and symptomatic HD group; $3=$ Significant difference $(p<.05)$ between the prodromal HD group and symptomatic HD group. $a=$ tests on which higher score is better; $\mathrm{b}=$ test on which lower score is better.

need to locate a target $(\mathrm{T})$ among 10 distractor stimuli (L). The target stimulus was rotated either $90^{\circ}$ or $270^{\circ}$ and the distractor stimuli were rotated either $0^{\circ}, 90^{\circ}, 180^{\circ}, 270^{\circ}$. The stimulus size was $0.81^{\circ} \times 0.81^{\circ}$. After instructions were given, subjects were asked to place their chin in a chinrest that was positioned $50 \mathrm{~cm}$ from the computer screen. Subjects were instructed to locate the target and indicate the direction of rotation of the target as quickly as possible by pressing one of two buttons of a response box. The experiment contained 16 blocks of 24 trials, resulting in 384 trials in total. In half of these trials, the spatial configuration (i.e., the positions) of the distractor and target stimuli were repeated. These trials are called "Repeated trials". Importantly, the direction of rotation of the targets in these trials was always randomly defined to prevent motor learning. In the other half of the trials, the positions of the stimuli and their direction of rotation were always randomly defined. These trials are called "New trials". Each block contained 12 Repeated trials and $12 \mathrm{New}$ trials. Each of the 12 Repeated trials contained a different spatial configuration that was repeated once per block. Therefore, each Repeated spatial configuration was shown once during each of the 16 blocks and thus repeated 16 times during the entire experiment. Before the start of the experiment 24 practice trials were given that did not contain any repeated contextual information.

\section{Recognition Memory Task}

To define whether subjects had noticed the repeated contextual information, three questions were asked immediately after finishing the experiment, namely: (1) "Did you notice anything during the experiment?" (2) "Did you notice that some of the configurations were repeated?" (3) "Did you try to remember the repeated configurations?". Subsequently, a recognition memory task was performed to define whether subjects had any conscious knowledge of the repeated spatial configuration. This recognition memory task contained 24 trials. During each trial, a spatial configuration was presented and subjects were instructed to indicate which of the trials had been repeated during the experiment by pressing one of two buttons on a response box. No time limit was used. Half of the trials contained repeated spatial configurations and half of the trials contained new spatial configurations.

\section{Statistical Analyses}

For statistical analysis, the 16 blocks were collapsed into 4 epochs of 4 blocks each (Figure 2). Trials in which an error (i.e., indicating the incorrect rotation of the target) was made were excluded (Controls: $1.1 \%$; prodromal HD participants: 0.8\%; symptomatic HD participants: $4.6 \%$ ) from analyses as 


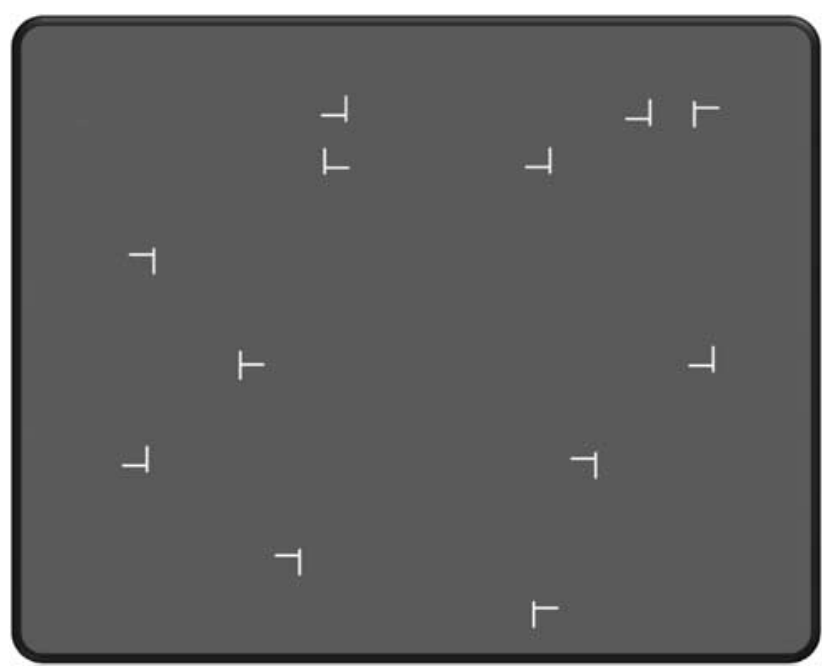

Fig. 1. A sample display of the contextual cueing task.

well as trials with a response time that took longer than $2 S D$ above the individual mean (Controls: $4.5 \%$; prodromal HD participants: $4.2 \%$; symptomatic HD participants: $4.4 \%$ ). A one-way ANOVA demonstrated an overall difference between the three groups for the number of errors $\left[F(2,44)=13.9 ; p<.01 ; \eta^{2}=0.39\right]$, indicating that the symptomatic HD participants made more errors than the controls, but not the prodromal HD participants $(p<.001)$. No group differences were found for the number of trials in which subjects responded too slow $[F(2,44)=0.2 ; p=.81]$.

Implicit learning is reflected by the difference in response times between the Repeated and New trials. This difference is called the contextual cueing effect. To define whether the contextual cueing effect was the same for the three groups, we performed a three-way repeated measures analyses, in which we included the between-subject variable Group (symptomatic HD participants, prodromal HD participants, controls) and the within-subject variables Configuration (repeated and new trials) and Epoch (1-4). As an index of the magnitude of the effects, the effect size $\eta^{2}$ was computed. Age, education level, and gender were included as covariates in the repeated measures analyses. For further interpretation of the results, it is important to realize that no significant age difference was found between the patient groups and the control group. Furthermore, previous research has shown that age does not affect implicit contextual learning (Howard, Dennis, Howard, Yankovich, \& Vaidya, 2004).

Since HD is a rare disease, the groups of participants that were included in this study were relatively small. A sample size analyses using the equation given by Rosner (2006), indicated that for the implicit contextual cueing task a sample size of 14 participants is needed to have sufficient power ( $\alpha=0.05$ and $\beta=0.8$ ). This means that the control and prodromal HD group have sufficient participants, but that the symptomatic HD group should have a few more. However, considering HD is an extremely rare disease and the fact that patients in an early stage have several symptoms that complicate participation, we were unable to increase the sample size. However, we have repeated the same analyses, now using only two groups, namely the control and HD group. The latter included both prodromal and symptomatic HD participants. Since these groups were now matched for age, education level, and gender, we did not include any covariates.

\section{RESULTS}

\section{Neuropsychological Tests}

To define whether the prodromal and symptomatic HD participants had cognitive impairments we applied an extensive battery of neuropsychological tasks. The results of these tests are reported in Table 1. Separate one-way ANOVAs were used to analyze the results of the different neuropsychological tests, comparing the symptomatic and prodromal HD group with the control subjects. When necessary we performed Dunnett post-hoc tests to compare the prodromal HD participants with the controls and the symptomatic HD participants with the controls. We found a significant Group effect for all tasks $(p \mathrm{~s}<.05)$, except for the Benton Visual Form Discrimination Test $[F(2,44)=2.8 ; p=.07]$. Post-hoc analyses indicated
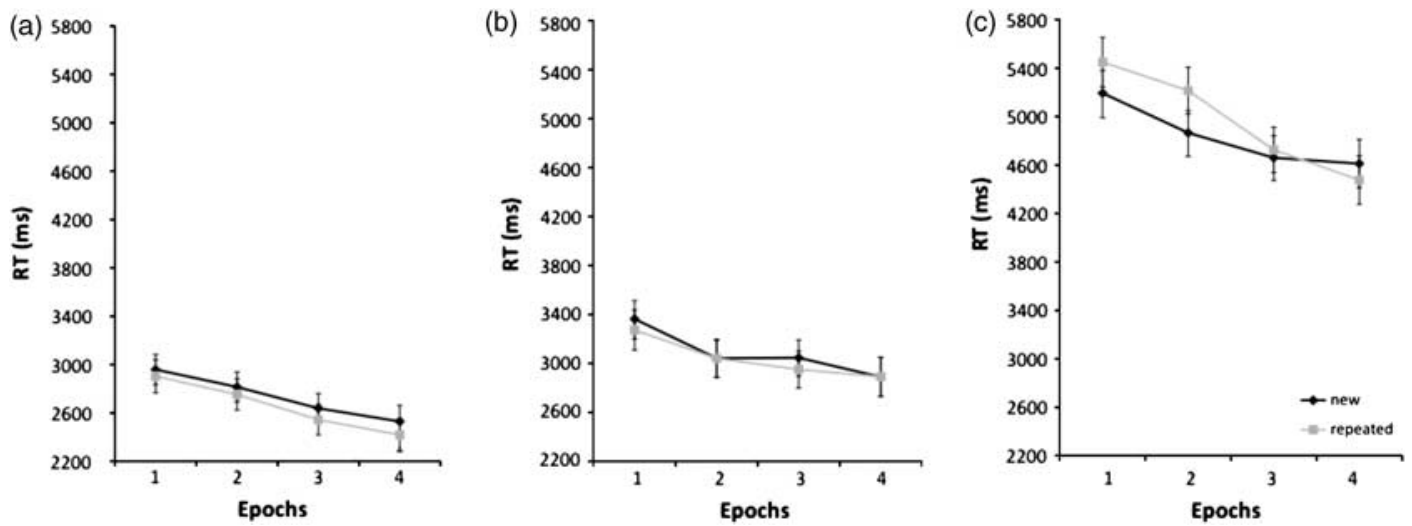

Fig. 2. Mean reaction times and standard errors for the Repeated and New trials separately as a function of Epoch (1-4). The three figures refer to the (a) control group; (b) prodromal HD group; (c) symptomatic HD group. 
that the symptomatic HD participants performed significantly worse than the control subjects on all tasks $(p<.05)$, whereas no significant difference was found between the prodromal HD participants and the control groups on any of the tasks. Symptomatic HD participants performed worse than prodromal HD participants on all tasks $(p<.05)$, except the Rey Auditory Verbal Learning Test and the Vocabulary subtest of the WAIS III.

\section{Implicit Contextual Learning Task}

The repeated measures analyses with the three groups (prodromal HD, symptomatic HD and controls) demonstrated a significant main effect for Group $[F(2,41)=41.7$; $\left.p<.001 ; \eta^{2}=0.67\right]$, indicating a difference in overall response times between the three groups. Contrast analyses indicated that HD participants $(p<.001)$ as well as the prodromal HD $(p<.05)$ participants responded significantly slower than the control subjects. Symptomatic HD participants are also slower than the prodromal HD participants $(p<.001)$. A significant main effect was also found for Epoch $\left[F(3,123)=2.9 ; p<.05 ; \eta^{2}=0.06\right]$, indicating that participants responded faster at the end of the experiment than at the beginning, reflecting visuomotor learning. The extent of learning was the same for the three groups of subjects, as was reflected by the nonsignificant interaction for Group $\times$ Epoch $[F(6,123)=2.1 ; p=.058]$. Importantly, a significant Group $\times$ Configuration effect was found $\left[F(2,41)=3.8 ; p<.05 ; \eta^{2}=0.16\right]$, demonstrating a difference in the extent of learning for the three groups. No three-way interaction was found $[F(6,123)=0.8 ; p=.61]$.

To understand which of the groups showed a significant contextual cueing effect, we performed separate repeated measures analyses for each Group, including the within-subject variable Configuration. For the control subjects, a contextual cueing effect was found that was reflected by a significant main effect for Configuration $\left[F(1,21)=9.3 ; p<.01 ; \eta^{2}=0.31\right]$. This demonstrates that within the control group response times were faster for the Repeated trials than for the New trials. When the same repeated measures analyses were performed for the prodromal $[F(1,15)=0.7 ; p=.72]$ and early-stage Huntington's disease participants $[F(1,8)=2.4 ; p=.16]$, no significant effect of Configuration was found, indicating that there was no difference in response times between the Repeated and New trials within the clinical groups.

Considering the small sample sizes of the patient groups, in particular the symptomatic HD group, we have repeated the previous analyses including only two groups, namely HD participants (including both prodromal and symptomatic participants) and the controls. We found a significant interaction effect for Group $\times$ Configuration $[F(1,45)=4.2$; $p<.05 ; \eta^{2}=0.09$ ], indicating a difference in contextual learning between the HD participants and the control subjects. Subsequently, we performed the two-way repeated measures analyses separately for the HD participants, including the within-subject variable Configuration. We did not find a significant effect for Configuration $[F(1,24)=0.2]$.
This confirms previous results indicating that HD participants are impaired on the implicit contextual cueing task.

\section{Recognition Memory Task}

To define whether subjects were aware of the repeated contextual information, we asked three questions after finishing the implicit contextual cueing task. Only one prodromal HD participant and one control subject indicated to have noticed the repeated contextual information (Question 1). Five prodromal HD participants and three control subjects indicated to have noted the repetitions when specifically asked about the contextual information (Question 2). Only one control subject indicated to have tried to memorize the repeated contextual information. For two control subjects, responses were missing.

To further define whether subjects had any conscious awareness of the repeated information, we tested all subjects with a recognition memory task. We found no significant difference between the number of correct responses on the recognition memory task and chance level for the controls, prodromal HD group, and HD participants $(\mathrm{t}<2.1)$. This indicates that subjects did not have any explicit knowledge of the repeated contextual information, thereby confirming the implicit nature of the contextual cueing task.

\section{DISCUSSION}

In the current study we tested participants with prodromal and symptomatic Huntington's disease using an implicit contextual cueing task using visuospatial information. The ability to learn contextual information and use this information to guide attention to a target is reflected in the reduction in response times for the trials with repeated contextual information in comparison to those trials with new contextual information. In our study, such a contextual cueing effect was found for the healthy control subjects, but not for the symptomatic or prodromal HD participants. This indicates that both prodromal and symptomatic HD patients are impaired on a spatial-based implicit contextual cueing task. These results are in line with previous studies that have shown deficits for different types of implicit learning in prodromal HD participants (Gabrieli et al., 1997; Ghilardi et al., 2008; Heindel et al., 1989; Kim et al., 2004).

Furthermore, the results of the current study are similar to previous findings in Parkinson's disease, another neurodegenerative disease affecting the basal ganglia, where we also observed an implicit learning deficit (Van Asselen et al., 2009). Together, these results suggest that the basal ganglia might play a role in implicit contextual learning. We cannot rule out, however, that abnormal function or connectivity of other brain regions affected by HD underlie this specific impairment. That is, in both Parkinson's and Huntington's disease, cortical areas such as the frontal lobes are also affected (Wolf, Vasic, Schönfeldt-Lecuona, Landwehrmeyer, \& Ecker, 2007). However, in our previous study, we tested Parkinson's disease patients in an early stage of the disease, 
in which the damage is largely confined to the basal ganglia. In the current study, we tested both prodromal HD patients and HD patients in an early stage of the disease. The finding that both patient groups were impaired strengthens the idea that damage to the basal ganglia might have caused the learning impairments. Furthermore, the medial temporal lobes, which also have an important role in implicit contextual learning (Chun \& Phelps, 1999; Greene et al., 2007; Manns \& Squire, 2001; Preston \& Gabrieli, 2008), are not affected in HD patients (Halliday et al., 1998). Finally, it should be noted that whereas patients with Huntington's disease and Parkinson's disease are impaired on an implicit contextual cueing task, patients with corticobasal syndrome are not (Negash et al., 2007), even though this is also a neurodegenerative disease that affects the basal ganglia. Subtle differences in disease pathology in Parkinson's disease, Huntington's disease, and corticobasal syndrome might explain the differences in impairment. Future studies should use neuroimaging techniques to define the exact subcortical areas that are involved.

To test overall cognitive functioning we have included a large battery of neuropsychological tests in the current study. Results of these tests indicated that the symptomatic HD participants were impaired on a wide range of tasks that were aimed at testing memory, executive functioning and visual perception. In contrast, prodromal HD participants were not impaired on any of these tasks. Although previous studies have found some cognitive functions to decline before onset of the disease, others indicated no impairments or suggested that the standard neuropsychological tests were not sensitive (Witjes-Ané et al., 2007). Indeed, in the current study we found that the prodromal HD participants were not impaired on any of the standard neuropsychological tasks, but were impaired on the implicit contextual cueing task. This suggests that the deficit in implicit learning is independent from general cognitive decline. In contrast, our findings that the symptomatic HD participants demonstrate a more general cognitive impairment affecting a wide variety of functions, thereby confirming previous studies (Lemiere et al., 2004). This is in line with the fact that they have more extensive damage to other cortical and subcortical brain areas.

It should be noted that Huntington's disease is a rare disorder, making it difficult to include a large group of patients, in particular when strict inclusion criteria are used and a distinction is being made between prodromal HD patients and symptomatic HD patients in an early stage of the disease. Analysis of sample size determined that the prodromal HD group is large enough, however, it could still be argued that the nonsignificant effect for the symptomatic HD participants is due to the small number of subjects that were included. For that reason, we have analyzed the data using only two groups, namely the HD participants (prodromal and symptomatic participants) and the control subjects. In this case, the HD participant group is even larger than the control group. The results of these analyses confirmed no implicit contextual learning in HD participants. Considering the small sample sizes of the patient groups, it is important to emphasize the need for replication to confirm our conclusions on implicit contextual learning deficits in HD.

In conclusion, we found that not only symptomatic HD participants, but also prodromal HD participants are impaired on a spatial based implicit contextual learning task. These results suggest that the basal ganglia might be involved in implicit contextual learning.

\section{ACKNOWLEDGMENTS}

The authors declare that the research described in this manuscript was conducted in the absence of any commercial or financial relationships that could be construed as a potential conflict of interest. The information in this manuscript and the manuscript itself has never been published either electronically or in print. This research was supported by a grant from the BIAL Foundation ( $\left.{ }^{\circ} 73 / 06\right)$ and the Portuguese Foundation for Science and Technology/COMPETE/FEDER (project PTDC/PSI-PCO/108208/2008 to MVA and PTDC/PSI/67381/2006 to $\mathrm{MCB})$.

\section{REFERENCES}

Beck, A.T., Ward, C.H., Mendelson, M., Mock, J., \& Erbaugh, J. (1961). An inventory for measuring depression. Archives of General Psychiatry, 4, 561-571.

Benton, A.L. (1974). Revised visual retention test (4th ed.). New York: Psychological Corporation.

Benton, A.L., Hamsher, K., Varney, N.R., \& Spreen, O. (1983). Contributions to neuropsychological assessment: A clinical manual. New York: Oxford University Press.

Berch, D.B., Krikorian, R., \& Huha, E.H. (1998). The Corsi BlockTapping Task: Methodological and theoretical considerations. Brain and Cognition, 38, 317-338.

Cavaco, S., Pinto, C., Gonçalves, A., Gomes, F., Pereira, A., \& Malaquias, C. (2008). Auditory Verbal Learning Test: Dados normativos dos 21 aos 65 anos [Norms for 21-65 years old]. Psychologica, 49, 208-221.

Chun, M.M. (2000). Contextual cueing of visual attention. Trends in Cognitive Sciences, 4(5), 170-178.

Chun, M.M., \& Jiang, Y. (1998). Contextual cueing: Implicit learning and memory of visual context guides spatial attention. Cognitive Psychology, 36, 28-71.

Chun, M.M., \& Phelps, E.A. (1999). Memory deficits for implicit contextual information in amnesic subjects with hippocampal damage. Nature Neuroscience, 2(9), 844-847.

Farrow, M., Churchyard, A., Chua, P., Bradshaw, J.L., Chiu, E., \& Georgiou-Karistianis, N. (2007). Attention, inhibition, and proximity to clinical onset in preclinical mutation carriers for Huntington's disease. Journal of Clinical and Experimental Neuropsychology, 29(3), 235-246.

Gabrieli, J.D.E., Stebbins, G.T., Singh, J., Willingham, D.B., \& Goetz, C.G. (1997). Intact mirror-tracing and impaired rotarypursuit skill learning in patients with Huntington's disease: Evidence for dissociable memory systems in skill learning. Neuropsychology, 11(2), 272-281.

Ghilardi, M.F., Silvestri, G., Feigin, A., Mattis, P., Zgaljardic, D., Moisello, C., ... Eidelberg, D. (2008). Implicit and explicit aspects of sequence learning in pre-symptomatic Huntington's disease. Parkinsonism \& Related Disorders, 14(6), 457-464.

Greene, J.A., Gross, W.L., Elsinger, C.L., \& Rao, S.M. (2007). Hippocampal differentiation without recognition: An fMRI 
analysis of the contextual cueing task. Learning \& Memory, 10, 548-553.

Gómez-Ansón, B., Alegret, M., Muñoz, E., Monté, G.C., Alayrach, E., ... Tolosa, E. (2009). Prefrontal cortex volume reduction on MRI in preclinical Huntington's disease relates to visuomotor performance and CAG number. Parkinsonism \& Related Disorders, 14(3), 213-219.

Halliday, G.M., McRitchie, D.A., Macdonald, V., Double, K.L., Trent, R.J., \& McCusker, E. (1998). Regional specificity of brain atrophy in Huntington's disease. Experimental Neurology, 154(2), 663-672.

Harris, G.J., Codori, A.M., Lewis, R.F., Schmidt, E., Bedi, A., \& Brandt, J. (1999). Reduced basal ganglia blood flow and volume in pre-symptomatic, gene-tested persons at-risk for Huntington's disease. Brain, 122, 1667-1678.

Heindel, W.C., Salmon, D.P., Shults, C.W., Walicke, P.A., \& Butters, N. (1989). Neuropsychological evidence for multiple implicit memory systems: A comparison of Alzheimer's, Huntington's and Parkinson's disease patients. The Journal of Neuroscience, 92, 582-587.

Howard, J.H., Dennis, N.A., Howard, D.V., Yankovich, H., \& Vaidya, H.Y. (2004). Implicit spatial contextual learning in healthy aging. Neuropsychology, 18(1), 124-134.

Kessels, R.P., Van Zandvoort, M.J., Postma, A., Kappelle, L.J., \& De Haan, E.H. (2000). The Corsi Block-Tapping Task: Standardization and normative data. Applied Neuropsychology, 7, 252-258.

Kim, J.S., Reading, S.A., Brashers-Krug, T., Calhoun, V.D., Ross, C.A., \& Pearlson, G.D. (2004). Functional MRI study of a serial reaction time task in Huntington's disease. Psychiatry Research, 131, 23-30.

Kirkwood, S.C., Siemers, E., Hodes, M.E., Conneally, P.M., Christian, J.C., \& Foroud, T. (2000). Subtle changes among presymptomatic carriers of the Huntington's disease gene. Journal of Neurology, Neurosurgery, and Psychiatry, 69, 773-779.

Kirkwood, S.C., Siemers, E., Stout, J.C., Hodes, M.E., Conneally, P.M., Christian, J.C., \& Foroud, T. (1999). Longitudinal cognitive and motor changes among presymptomatic Huntington disease gene carriers. Archives of Neurology, 56(5), 563-568.

Lawrence, A.D., Hodge, J.R., Rosser, A.E., Kershaw, A., FrenchConstant, C., Rubinsztein, D.C., ... Sahakian, B.J. (1998). Evidence for specific cognitive deficits in preclinical Huntington's disease. Brain, 121, 1329-1341.

Lemiere, J., Decruyenaere, M., Evers-Kieboms, G.E., Vandenbussche, E., \& Dom, R. (2004). Cognitive changes in patients with Huntington's disease (HD) and asymptomatic carriers of the HD mutation. Journal of Neurology, 251, 935-942.

Manns, J.R., \& Squire, L.R. (2001). Perceptual learning, awareness, and the hippocampus. Hippocampus, 11, 776-782.

Mattis, S., Jurica, P.J., \& Leitten, C.L. (2002). Dementia Rating Scale (DRS-2). Lutz, FL: Psychological Assessment Resources.

Negash, S., Boeve, B.F., Geda, Y.E., Smith, G.E., Knopman, D.S., Ivnik, R.J., ... Petersen, R.C. (2007). Neurocase, 13, 133-143.

Nopoulos, P.C., Aylward, E.H., Ross, C.A., Johnson, H.J., Magnotta, V.A., Juhl, A.R., ... Paulsen, J.S.; PREDICT-HD Investigators Coordinators of Huntington Study Group. (2010). Cerebral cortex structure in prodromal Huntington disease. Neurobiology of Disease, 40(3), 544-554.

Oldfield, R.C. (1971). The assessment and analysis of handedness: The Edinburgh inventory. Neuropsychologia, 9(1), 97-113.

Osterrieth, P.A. (1944). "Filetest de copie d'une figure complex: Contribution a l' étude de la perception et de la memoire [The test of copying a complex figure: A contribution to the study of perception and memory]". Archives de Psychologie, 30, 286-356.

Paulsen, J.S., Nopoulos, P.C., Aylward, E., Ross, C.A., Johnson, H., Magnotta, V.A., ... Nance, M.; PREDICT-HD Investigators and Coordinators of the Huntington's Study Group. (2010). Striatal and white matter predictors of estimated diagnosis for Huntington disease. Brain Research Bulletin, 31(82), 201-207.

Preston, A.R., \& Gabrieli, J.D. (2008). Dissociation between explicit memory and configural memory in the human medial temporal lobe. Cerebral Cortex, 18, 2192-2207.

Raven, J.C., Raven, J., \& Court, J.H. (1993). Manual for Raven's progressive matrices and vocabulary scales. Oxford: Oxford Psychologists Press.

Rey, A. (1964). L'examen clinique en psychologie [Clinical assessment in psychology]. Paris: Presses Universitaires de France.

Rey, A. (1941). L'examen psychologique dans les cas d'encephalopathie traumatique. Arch Psychol, 28, 286-340.

Robins Wahlin, T.B., Lundin, A., \& Dear, K. (2007). Early cognitive deficits in Swedish gene carriers of Huntington's disease. Neuropsychology, 21(1), 31-44.

Rosner, B. (2006). Fundamentals of bioststistics. Pacific Grove, CA: Duxbury Press.

Snowden, J.S., Craufurd, D., Thompson, J., \& Neary, D. (2002). Psychomotor, executive and memory function in preclinical Huntington's disease. Journal of clinical and experimental neuropsychology, 24(2), 133-145.

Stout, J.C., Paulsen, J.S., Queller, S., Solomon, A.C., Whitlock, K.B., Campbell, J.C., ... Aylward, E.H. (2011). Neurocognitive signs in prodromal Huntington disease. Neuropsychology, 25, $1-14$.

Tabrizi, S.J., Langbehn, D.R., Leavitt, B.R., Roos, R.A., Durr, A., Craufurd, D., ... Stout, J.C., \& TRACK-HD Investigators. (2009). Biological and clinical manifestations of Huntington's disease in the longitudinal TRACK-HD study: Cross-sectional analysis of baseline data. The Lancet, 8, 791-801.

Tabrizi, S.J., Scahill, R.I., Durr, A., Roos, R.A., Leavitt, B.R., Jones, R., ... Stout, J.C., \& TRACK-HD Investigators. (2011). Biological and clinical changes in premanifest and early stage Huntington's disease in the TRACK-HD study: The 12-month longitudinal analysis. The Lancet, 10, 31-42.

The Huntington's Disease Collaborative Research Group. (1993). A novel gene containing a trinucleotide repeat that is expanded and unstable on Huntington's disease chromosomes. Cell, 72, 971-983.

Thieben, M.J., Duggins, A.J., Good, C.D., Gomes, L., Mahant, N., Richards, F., ... Frackowiak, R.S. (2002). The distribution of structural neuropathology in pre-clinical Huntington's disease. Brain, 125, 1815-1828.

Van Asselen, M., Almeida, I., André, R., Januário, C., Freire Gonçalves, A., \& Castelo-Branco, M. (2009). The role of the basal ganglia in implicit contextual learning: A study of Parkinson's disease. Neuropsychologia, 47, 1269-1273.

Vaz Serra, A., \& Pio Abreu, J.L. (1973a). Aferição dos quadros clínicos depressivos. I - Ensaio de aplicação do "Inventário Depressivo de Beck" a uma amostra portuguesa de doentes deprimidos. Coimbra Médica, XX, 623-644.

Vaz Serra, A., \& Pio Abreu, J.L. (1973b). Aferição dos quadros clínicos depressivos. II - Estudo preliminar de novos agrupamentos sintomatológicos para complemento do "Inventário Depressivo de Beck”. Coimbra Médica, XX, 713-736. 
Wechsler, D. (1997). WAIS-III: Administration and scoring manual. San Antonio, TX: The Psychological Corporation.

Wechsler, D. (2008). WAIS-III: Escala de Inteligência de Wechlser para Adultos -3. ${ }^{\underline{a}}$ edição: Manual [Wechsler Adult Intelligence Scale (WAIS-III)]. Lisboa: Cegoc.

Witjes-Ané, M.N., Mertens, B., van Vugt, J.P., Bachoud-Lévi, A.C., van Ommen, G.J., \& Roos, R.A. (2007). Longitudinal Evaluation of "Presymptomatic" Carriers of Huntington's Disease. The Journal of Neuropsychiatry and Clinical Neuroscience, 19(3), 310-317.

Wolf, R.C., Vasic, N., Schönfeldt-Lecuona, C., Landwehrmeyer, G.B., \& Ecker, D. (2007). Dorsolateral prefrontal cortex dysfunction in presymptomatic Huntington's disease: Evidence from event-related fMRI. Brain, 130, 2845-2857. 